\title{
A GRADE Working Group approach for rating the quality of treatment effect estimates from network meta-analysis
}

In table 1 of this Research Methods and Reporting article (BMJ 2014;349:g5630, doi:10.1136/bmj.g5630), the authors correctly state for the comparison of vitamin D plus calcium versus risedronate that the quality of evidence for the network meta-analysis (NMA) estimate is low. This is based on the rule that where both direct and indirect evidence are available, the higher of the two quality ratings should be used, which in this case is a low rating. However, in the penultimate paragraph of the section "Steps 3 and 4: Presenting and rating of quality of NMA effect estimates," the authors wrote: "For the comparison of vitamin D plus calcium versus risedronate, direct evidence had very low confidence rating and contributed substantially more to the NMA estimate than indirect evidence; therefore, the quality rating for the NMA estimate was also very low."

The authors would like to correct this sentence to the following: "For the comparison of vitamin D plus calcium versus risedronate, direct evidence had a very low confidence rating and indirect evidence had a low confidence rating; therefore, the quality rating for the NMA estimate was low."

Cite this as: BMJ 2015;350:h3326

๑๑ BMJ Publishing Group Ltd 2015 